\title{
Antiobesogenic effects of central GIPR antagonism
}

\author{
Jessica T.Y. Yue ${ }^{1}$ and Tony K.T. Lam ${ }^{2,3,4,5}$ \\ 'Department of Physiology, University of Alberta, Edmonton, Alberta, Canada. ${ }^{2}$ Toronto General Hospital Research Institute, University Health Network (UHN), Toronto, Ontario, Canada. ${ }^{3}$ Department of \\ Physiology, ${ }^{4}$ Department of Medicine, and ${ }^{5}$ Banting and Best Diabetes Centre, University of Toronto, Toronto, Ontario, Canada.
}

\begin{abstract}
Developing effective treatments for obesity and related metabolic disease remains a challenge. One logical strategy targets the appetite-regulating actions of gut hormones such as incretins. One of these incretins, glucosedependent insulinotropic polypeptide (GIP), has garnered much attention as a potential target: however, whether it is beneficial to boost or block the action of CIP to promote weight loss remains an unresolved question. In this issue of the $J C I$, Kaneko and colleagues show that antagonizing GIP signaling in the CNS enhances the weight-reducing effects of leptin in rodents with diet-induced obesity. The authors posit that an increase in circulating intestinally derived GIP, as a consequence of overnutrition, acts in the brain to impair hypothalamic leptin action, resulting in increased food intake and body weight gain. This research advances the idea that multiple GIP signaling pathways and mechanisms exist in the obese state and offers intriguing insights into the antiobesogenic consequences of antagonizing brain GIP action.
\end{abstract}

\section{A therapeutic benefit from GIP deficiency}

Obesity remains a multifaceted disease, the management of which continues to be complex yet necessary to circumvent its related adverse health outcomes. Effective and safe antiobesity medications remain elusive, and this unmet need is heightened as a result of the increasing global prevalence of obesity and obesity-related metabolic diseases. Agonists of receptors for gut-derived hormones, including those based on incretins, are prescribed to treat type 2 diabetes and obesity (1). Analogs for glucagon-like peptide 1 (GLP-1) such as liraglutide are more stable in plasma than native GLP-1 and potently lower glycemia, promote satiety, and reduce body weight. Another incretin, glucose-dependent insulinotropic polypeptide (GIP), is released postprandially by enteroendocrine cells, binds to the GIP receptor (GIPR) on pancreatic $\beta$ cells, and stimulates insulin secretion (2). In contrast to GLP-1, the therapeutic importance of GIP analogs as treatment for diabetes and obesity has gained little traction, largely due to insufficient insulinotropic efficacy in hyperglycemia (3) and obesity (4) and its lack of effect on satiety and resting energy expenditure in humans (5).

Interestingly, obesity and high-fat diet (HFD) feeding in rodents and humans are associated with increased secretion and circulating levels of GIP (6-8). Moreover, life-long reduction of GIP activity in Gipr-knockout mice (9), loss of GIP-secreting K cells (10), or genetic loss of GIP (11) all protect against diet-induced obesity, indicating a therapeutic benefit from GIP deficiency. Intriguingly, preclinical and clinical evidence indicates that a combination of GLP-1 and GIP signaling decreases glucose and body weight beyond what is seen with GLP-1 signaling alone (12-14). Hence, a better understanding of the site of action

Related Article: p. 3786

Conflict of interest: The authors have declared that no conflict of interest exists.

Copyright: @ 2019, American Society for Clinical Investigation.

Reference information: / Clin Invest. 2019;129(9):3532-3535. https://doi.org/10.1172/JCI130755.

and mechanisms involved in these weightreducing effects, as well as the time-dependent effect of GIPR manipulation, could help guide therapeutic development.

In this issue of the JCI, Kaneko and colleagues (15) provide compelling evidence of a mechanism that drives weight reduction in response to inhibition of GIPR signaling within the brain (Figure 1). The authors administered a previously characterized (16) neutralizing monoclonal antibody (Gipg013), which specifically and potently antagonizes GIPR, directly into the rodent brain. Blocking GIP signaling within the brain for two weeks lowered food intake and body weight, which was associated with reductions in fat mass, in obese mice compared with obese mice that received control antibody infusions. Conversely, these alterations did not occur in lean mice in which circulating GIP presumably remains normal. Since blocking central GIPR in the brain failed to lower food intake and body weight in leptin-deficient $o b / o b$ mice, the authors conclude that GIPR-related antiobesity effects require intact leptin signaling. Of note, inhibition of GIPR in the brain alone reduced body weight and suppressed appetite in dietinduced obese mice, but when combined with GLP-1R agonism, this inhibition failed to further potentiate decreases in body weight and food intake. Hence, in the brain, GIPR blockade requires intact leptin signaling to exert antiobesity effects. However, peripherally, GIPR inhibition effects act independently of leptin. Blocking GIPR activity, either via whole-body genetic deletion in diet-induced obese mice, or in $o b / o b$ mice, exerts antiobesogenic effects (9). Moreover, whereas blocking brain GIPR confers no additional reductions in body weight or food intake when combined with GLP-1R stimulation, blocking peripheral GIP signaling while stimulating GLP-1R enhances antiobesogenicity (ref. 17 and Figure 1). Taken together, GIPR antagonism in the brain and the periphery appears to have distinct mechanisms for regulating body weight in obesity. 
A
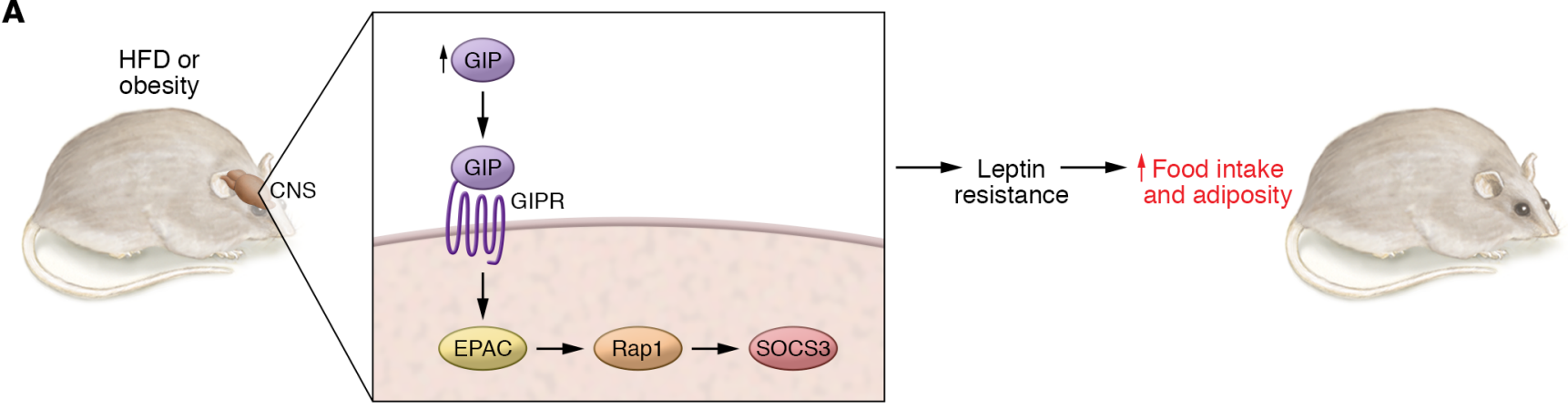

B
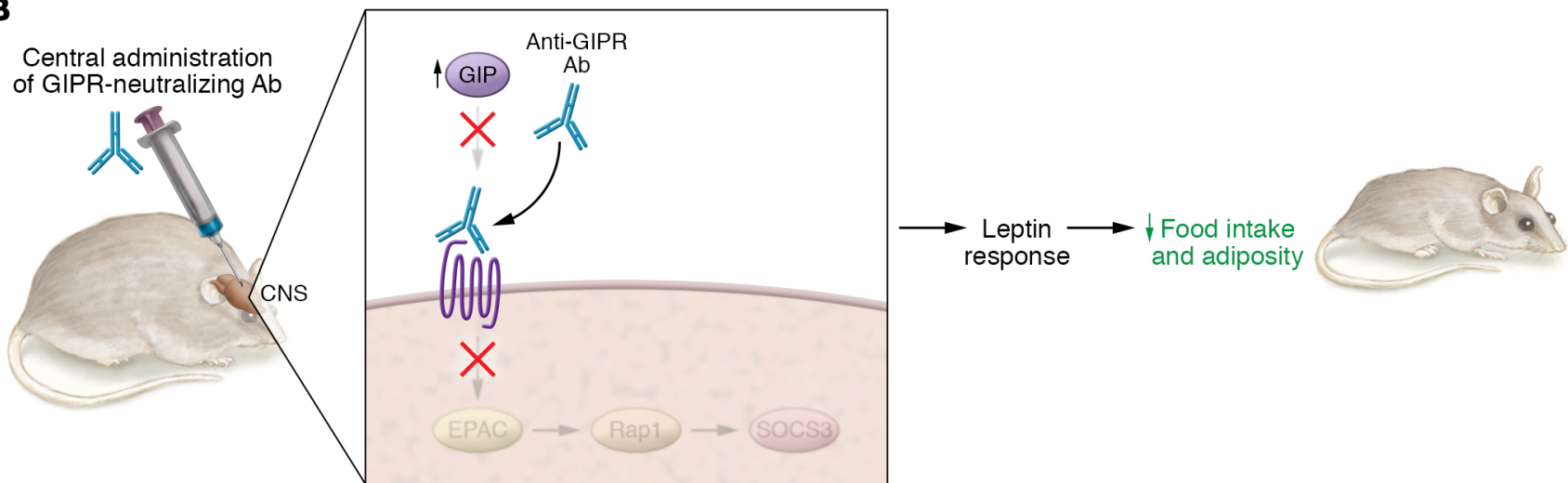

\section{C} Peripheral administration
of GIPR-neutralizing Ab plus GLP-1 agonist
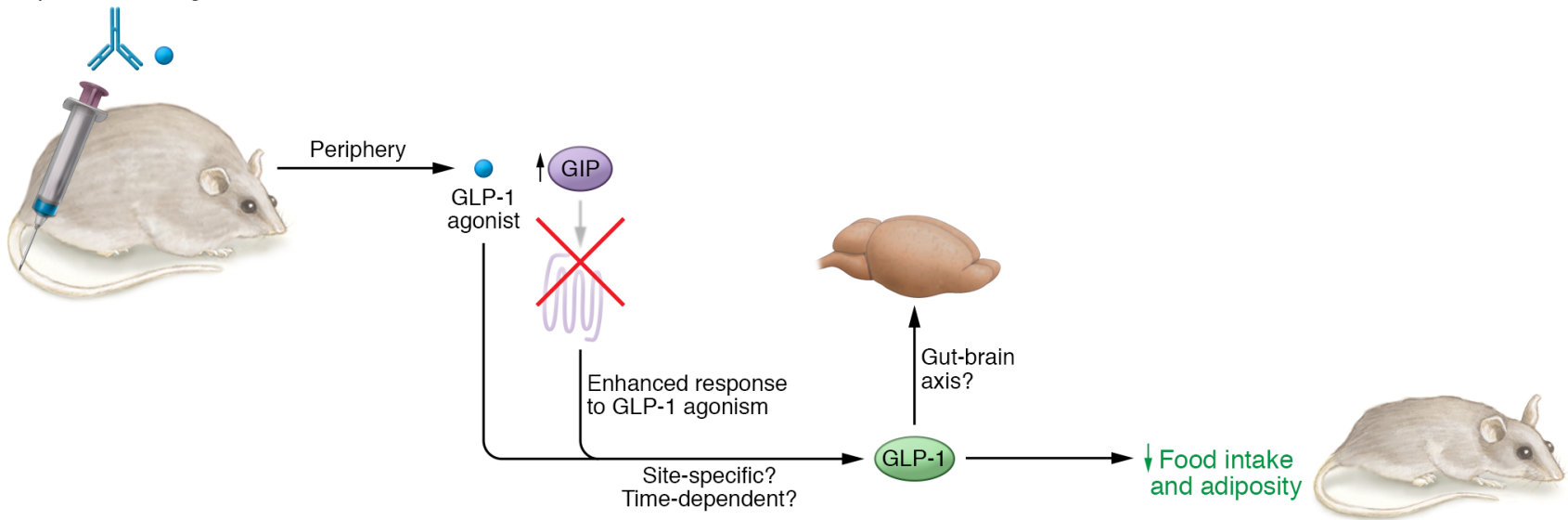

Figure 1. Effects of targeting GIP in the CNS and periphery. HFD feeding and/or obesity increases circulating GIP levels. Increased GIP levels in the CNS (i.e., hypothalamus) activates GIPR and stimulates the GIPR/EPAC/Rap1/SOCS3 pathway, which induces leptin resistance, hyperphagia, and obesity. Central blockade of GIPR, via delivery of a GIPR-neutralizing antibody, consequently enhances leptin action to lower food intake and obesity. Peripheral blockade of GIPR in parallel with GLP-1 agonism enhances GLP-1 action to lower food intake and obesity in a leptin-independent manner. Future studies are needed to investigate whether CNS GIPR signaling alters insulin sensitivity and glucose and lipid homeostasis, and whether the coagonistic metabolic beneficial effects of GIP and GLP-1 are different from those observed with GIPR antagonists due to differences in tissue specificity and/or time-dependent effects.

\section{The contribution of GIP} signaling to leptin resistance Administering leptin directly to the nervous system reduces food intake and weight gain in lean WT mice, however, leptin fails to lower body weight in mice fed a HFD. To underscore the pivotal role of central GIPR signaling in affecting neu- ral leptin action, Kaneko and colleagues demonstrated that genetic Gipr deficiency markedly enhanced the anorexic and weight-lowering effects of leptin in HFDfed mice. As expected, in lean mice, in which neural leptin signaling is intact and GIP levels presumably remain normal, Gipr deficiency does not affect the meta- bolic effects imposed by leptin. To further support the inhibitory role of GIPR activation in enhancing hypothalamic leptin action, Kaneko et al. investigated intracellular SOCS3 levels in the hypothalamus. Obesity-related increases in SOCS3 expression were reversed by chronic, two-week neural GIPR blockade (via the 
GIPR antagonist Gipg013). Conversely, administering GIP into the brain negated leptin-related weight-lowering effects and disabled leptin-dependent phosphorylated STAT3 (p-STAT3) transduction, while increasing hypothalamic Socs3. Collectively, these findings highlight the idea that brain GIPR inhibition alone in obese mice prevents excessive endogenous GIP action that otherwise impairs central leptin signaling and anorexic effects. Given that local hypothalamic Gip mRNA levels were low, future studies to determine the extent to which peripheral GIP crosses the bloodbrain barrier and/or increases in the hypothalamus in obesity could shed light on the relative importance of gut-derived GIP in impairing central energy homeostatic circuits governed by leptin action.

Kaneko et al. report that peripheral administration of exogenous GIP increases levels in the blood (mimicking the levels observed in obesity) as well as cerebrospinal fluid, suggesting that blood-brain GIP transport is related to the central actions of leptin on food intake and body weight regulation (15). Of note, the CNS effect of leptin on food intake and body weight in HFD-fed mice is only partially blocked by GIP; thus, central leptin resistance is not fully attributable to GIP and could have other sources. Leptin activates hypothalamic proopiomelanocortin (POMC) neurons to exert anorexic effects. As assessed through whole-cell patch clamps, GIP abolishes the effect of leptin on POMC neuronal firing. Future studies examining loss of function will clarify the role for POMC neurons in mediating GIP signaling effects on energy metabolism. Collectively, pharmacological and genetic manipulations in vivo, assessed by metabolic measurements of feeding and weight changes and immunohistochemical and molecular analyses, demonstrate that activation of GIPR induces hypothalamic leptin resistance.

How might GIP induce hypothalamic leptin resistance? GIP activates the GPCR GIPR to trigger intracellular cAMP signaling, which in turn activates both protein kinase A (PKA) and exchange protein directly activated by cAMP (EPAC). Kaneko et al. applied GIP to ex vivo brain slices, which suppressed the leptin mediator p-STAT in the presence and absence of PKA inhibition (15). Conversely, EPAC inhibition blocked leptin action, indicating that EPAC mediates GIP/GIPR signaling to inhibit hypothalamic leptin action (15). Furthermore, applying GIP (both in vivo and ex vivo) increased the active form of the small GTPase Rap1, a direct target of EPAC. This mechanism is analogous to the insulinotropic effects of incretins on $\beta$ cells that likewise require EPAC/Rap1 activation to stimulate insulin exocytosis (18). Importantly, EPAC activation alone induces leptin resistance (19), and neuronal Rap1, which is activated in diet-induced obesity, modulates leptin responsivity to regulate metabolism (20).

Notably, the work presented by Kaneko and colleagues shows that GIPR antagonism prevents HFD-induced increases in Rap1 activation (15). Support for the hypothesis that EPAC/Rap1 signaling is necessary for hypothalamic GIPR signaling to induce leptin resistance and obesity was obtained in mice with Rap1 deficiency in forebrain structures including hypothalamic nuclei (Rap1 $\left.{ }^{\Delta \mathrm{CNS}}\right)$. Whereas central GIP infusion increased hypothalamic Socs 3 expression and blunted leptinrelated weight-lowering effects in control mice, Rap $1^{\Delta \mathrm{CNS}}$ mice were protected from GIP-induced leptin resistance in the brain. Thus, Kaneko et al. provide compelling evidence that Rap1 activation in the brain is necessary for hypothalamic GIPR signaling to induce leptin resistance and obesity in rodents.

Overnutrition via HFD feeding elevates circulating GIP levels and consequently enhances CNS GIP-mediated activation of EPAC/Rap1 signaling; in turn, induced hypothalamic SOCS3 impairs leptin sensitivity, which promotes body weight gain and adiposity (Figure 1). Kaneko and colleagues manipulated CNS GIPR signaling, while peripheral GIPR signaling remained intact. Notably, in addition to the CNS effect, peripheral administration of anti-GIPR-antagonizing antibodies, which are likely restricted from the blood-brain barrier, also showed antiobesity effects. These peripheral, and potentially therapeutic, effects are seen in obese mice and obese nonhuman primates, in which weight loss is further enhanced when these antibodies are administered with GLP-1R agonists in a mechanism that is independent of the pancreatic $\beta$ cell GIPR (17).

\section{Conclusions}

Although elucidation of the underlying antiobesity effect of central GIPR signaling remains urgent, it is equally important to investigate the metabolic effect of peripheral GIPR signaling. The target tissues affected by peripheral administration of GIPR agonists or antagonists to regulate energy balance remain unknown. GIP stimulates lipid deposition in visceral adipose tissue and stimulates adipogenesis $(9,21)$; thus, GIPR antagonism in adipose tissue, for example, may help to improve insulin sensitivity and thereby beneficially affect body weight and satiety.

The vagus nerve relays postprandial signals from the gut to the brain, in part through GLP-1 signaling (22). It is not evident, however, that GIP acts directly within the vagal afferents $(22,23)$, which may also explain some of the divergent effects of the two incretin hormones on energy balance. Also of note is a recent randomized crossover study in which incretin hormones were infused to mimic elevated levels achieved during an oral glucose tolerance test in overweight/obese men to determine whether combined infusions of GIP and GLP-1 suppress appetite to a greater degree than does GLP-1 alone (24). GLP-1 infusions, but not GIP alone or GIPGLP-1 combination infusions, lower energy intake compared with that achieved with saline infusion controls. Rather, coinfusion of GIP and GLP-1 negates the appetite-suppressing effects of GLP-1 (24), which may support the findings of Kaneko et al. (15). Nonetheless, comparing and contrasting the food intake-regulatory effects of CNS versus peripheral GIPR signaling in rodents, nonhuman primates, and humans in obese, diabetic, and healthy conditions, as well as dissecting the time-dependent effects of GIPR inhibition and/or activation (Figure 1) should remain a priority in obesity research.

The results of Kaneko et al. require additional studies to further elucidate the causal actions of GIP in obesity and related diseases. An integral connection exists between hypothalamic leptin and insulin action, defects of which predispose to obesity and type 2 diabetes; and it remains to be seen whether CNS GIP signaling also affects CNS insulin sensitivity. Furthermore, given that CNS leptin and insulin action are involved not only in energy bal- 
ance but also the control of glucose and lipid homeostasis, investigations into the role of CNS GIP signaling in peripheral glucose and fat metabolism are needed to address the clinical relevance of targeting neural GIPR action (Figure 1).

As lasting and effective antiobesity medications necessitate continued use, it remains important to determine whether long-term antagonism of central GIPR action will be efficacious and free from side effects. Although bariatric surgery is most effective at achieving sustained weight reduction, safe and efficacious medication provides an alternative to surgical intervention. The research finding of Kaneko et al. (15) offers a promising drug target and potential pharmacotherapy for obesity.

\section{Acknowledgments}

JTYY is supported by the Canadian Institutes for Health Research (CIHR) (PJT378765), the Natural Sciences and Engineering Research Council of Canada (RGPIN-2016-06657), and the Canada Foundation for Innovation John R. Evans Leaders Fund (grant 37267), and is a Diabetes Canada Scholar (SC-5-16-5060-JY) and a Tier 2 Canada Research Chair in Brain Regulation of Metabolism at the University of Alberta. TKTL is supported by a CIHR Foundation Grant (FDN-143204), holds the John Kitson McIvor (1915-1942) Endowed Chair in Diabetes Research, and is a Tier 1 Canada Research Chair in Diabetes and Obesity at the Toronto General Hospital Research Institute and the University of Toronto.

Address correspondence to: Jessica Yue, 7-21 Medical Sciences Building, Department of Physiology, University of Alberta, Edmonton, Alberta, Canada T6G2H7. Phone: 780.248.5804; Email: jessica. yue@ualberta.ca. Or to: Tony Lam, MaRS Centre, 101 College Street, Toronto Medical Discovery Tower, 10th floor, Room
705, Toronto, Ontario, Canada M5G1L7. Phone: 416.581.7880; Email: tony.lam@ uhnres.utoronto.ca.

1. Bessesen DH, Van Gaal LF. Progress and challenges in anti-obesity pharmacotherapy. Lancet Diabetes Endocrinol. 2018;6(3):237-248.

2. Campbell JE, Drucker DJ. Pharmacology, physiology, and mechanisms of incretin hormone action. Cell Metab. 2013;17(6):819-837.

3. Nauck MA, Heimesaat MM, Orskov C, Holst JJ, Ebert R, Creutzfeldt W. Preserved incretin activity of glucagon-like peptide 1 [7-36 amide] but not of synthetic human gastric inhibitory polypeptide in patients with type-2 diabetes mellitus JClin Invest. 1993;91(1):301-307.

4. Calanna S, et al. Beta and alpha cell function in metabolically healthy but obese subjects: relationship with entero-insular axis. Obesity (Silver Spring). 2013;21(2):320-325.

5. Asmar M, et al. On the role of glucose-dependent insulintropic polypeptide in postprandial metabolism in humans. Am J Physiol Endocrinol Metab. 2010;298(3):E614-E621.

6. Creutzfeldt W, Ebert R, Willms B, Frerichs H, Brown JC. Gastric inhibitory polypeptide (GIP) and insulin in obesity: increased response to stimulation and defective feedback control of serum levels. Diabetologia. 1978;14(1):15-24.

7. Suzuki K, et al. Transcriptional regulatory factor X6 (Rfx6) increases gastric inhibitory polypeptide (GIP) expression in enteroendocrine $\mathrm{K}$-cells and is involved in GIP hypersecretion in high fat diet-induced obesity. J Biol Chem . 2013;288(3):1929-1938.

8. Brøns C, et al. Impact of short-term high-fat feeding on glucose and insulin metabolism in young healthy men. J Physiol (Lond). 2009;587(Pt 10):2387-2397.

9. Miyawaki K, et al. Inhibition of gastric inhibitory polypeptide signaling prevents obesity. Nat Med. 2002;8(7):738-742.

10. Althage MC, Ford EL, Wang S, Tso P, Polonsky KS, Wice BM. Targeted ablation of glucosedependent insulinotropic polypeptide-producing cells in transgenic mice reduces obesity and insulin resistance induced by a high fat diet. J Biol Chem. 2008;283(26):18365-18376.

11. Nasteska D, et al. Chronic reduction of GIP secretion alleviates obesity and insulin resistance under high-fat diet conditions. Diabetes. 2014;63(7):2332-2343.

12. Finan B, et al. Unimolecular dual incretins maximize metabolic benefits in rodents, monkeys, and humans. Sci Transl Med. 2013;5(209):209ra151.
13. Frias JP, et al. Efficacy and safety of LY3298176, a novel dual GIP and GLP-1 receptor agonist, in patients with type 2 diabetes: a randomised, placebo-controlled and active comparator-controlled phase 2 trial. Lancet. 2018;392(10160):2180-2193.

14. Mroz PA, et al. Optimized GIP analogs promote body weight lowering in mice through GIPR agonism not antagonism. Mol Metab. 2019;20:51-62.

15. Kaneko K, et al. Gut-derived GIP activates central Rap1 to impair neural leptin sensitivity during overnutrition. J Clin Invest. 2019;129(9):3786-3791.

16. Ravn P, et al. Structural and pharmacological characterization of novel potent and selective monoclonal antibody antagonists of glucosedependent insulinotropic polypeptide receptor. J Biol Chem. 2013;288(27):19760-19772.

17. Killion EA, et al. Anti-obesity effects of GIPR antagonists alone and in combination with GLP$1 \mathrm{R}$ agonists in preclinical models. Sci Transl Med. 2018;10(472):eaat3392.

18. Kang G, Chepurny OG, Holz GG. cAMPregulated guanine nucleotide exchange factor II (Epac2) mediates $\mathrm{Ca} 2+$-induced $\mathrm{Ca} 2+$ release in INS-1 pancreatic beta-cells. J Physiol (Lond) 2001;536(Pt 2):375-385

19. Fukuda M, Williams KW, Gautron L, Elmquist JK. Induction of leptin resistance by activation of cAMP-Epac signaling. Cell Metab. 2011;13(3):331-339.

20. Kaneko K, et al. Neuronal Rap1 regulates energy balance, glucose homeostasis, and leptin actions. Cell Rep. 2016;16(11):3003-3015.

21. Song DH, Getty-Kaushik L, Tseng E, Simon J, Corkey BE, Wolfe MM. Glucose-dependent insulinotropic polypeptide enhances adipocyte development and glucose uptake in part through Akt activation. Gastroenterology. 2007;133(6):1796-1805.

22. Egerod KL, et al. Profiling of G protein-coupled receptors in vagal afferents reveals novel gut-to-brain sensing mechanisms. Mol Metab 2018;12:62-75.

23. Nishizawa M, Nakabayashi H, Uchida K, Nakagawa A, Niijima $A$. The hepatic vagal nerve is receptive to incretin hormone glucagon-like peptide-1, but not to glucose-dependent insulinotropic polypeptide, in the portal vein. JAuton Nerv Syst. 1996;61(2):149-154.

24. Bergmann NC, et al. Effects of combined GIP and GLP-1 infusion on energy intake, appetite and energy expenditure in overweight/obese individuals: a randomised, crossover study. Dia betologia. 2019;62(4):665-675. 